\title{
Neurofeedback versus mindfulness on young football players' anxiety and performance
}

\author{
Seyed Mohammad Zadkhosh, Hassan Gharayagh Zandi, \\ Rasool Hemayattalab
}

Faculty of Physical Education and Sport Sciences, University of Tehran, Iran.

\begin{abstract}
Nowadays, in athletic world exercise and physical practice are not only tools to achieve peak performance. In high level of athletic activities there are not so differences based on skilfulness and psychological differences determinant winner or loser. Athletes have to compete in anxiety situations and in reaction to these situations are experiencing excitement. Mindfulness and Neurofeedback interventions are included interventions that are used in the field of psychology, particularly sport psychology that includes two disparate aspects of psychological interventions i.e. technological and traditional. Therefore in this study, we examined the effects of Mindfulness and Neurofeedback interventions on the level of Sport Anxiety and Athletic performance of young football players. In this study, 45 Iranian Football players were assigned to three groups. Athletes in group 1 received twelve-session, 30-minute alpha/theta neurofeedback training, in group 2 received twelvesession, 30-minutes mindfulness training and control group did not receive any intervention. Sport Anxiety Scale and Shoot performance test were used for quantitative comparisons. The results of MANOVA showed that improvement of athletic performance scores and reduction of anxiety scale were significant in both experimental groups. In addition, there were not significant differences between both experimental groups in athletic performance, but there were significantly different between mindfulness groups compared to the neurofeedback group in sport anxiety scale. According to these findings, we concluded the alpha/theta neurofeedback training and mindfulness meditation training are appropriate to increase athletic performance and decrease level of sport anxiety.
\end{abstract}

Keywords. Football player, mindfulness, neurofeedback, performance, sport anxiety.

\section{Introduction}

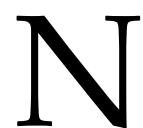

owadays, in sport world exercise and physical practice are not only tools to achieve peak performance; because in addition, physical, tactical abilities and proficiency, aptitude and personality characteristics are efficacious factors to athletic promotion (Martin et al., 2005). Jones et al. (2007) maintain that in high level of athletic activities there are not so differences based on skilfulness and psychological differences determinant winner or loser. Extensive studies have been done in the field of sport psychology skills training. Such trainings are to help athletes up to earned and maintain its high performance (Krane \& Williams, 2006). In this regard, in the study that took place by Orlick and Partington (1988) on the performance of successful and unsuccessful athletes in an equal level, the results indicated that factors that could cause these differences were psychological reasons which were includes attention, stress management and commitment to progress. In another study Gould et al. (2002) concluded that the most important psychological characteristics of elite athletes are the ability to cope and control their anxiety. Athletes have to compete in anxiety situations and in reaction to these situations are experiencing excitement (Martin et al., 2005). Prevalence anxiety factors such as bad refereeing during the match, bad environmental conditions, audiences' reaction during the match, desire to win at the cost away from athletic ethics, experience of injury, seeing rival fraud, possibility of fraud in order to win, reprimand player by coach and coach criticize during the match are inevitable the necessity of effective coping skills in order to preserve the mental health of athlete and achieve athletic success; because inability to deal with anxiety will be harmful for successful performance of athletes (Anshel et al., 2000).

Received: October 04, 2018 - Accepted: December 28, 2018 - Published: December 30, 2018

To cite this article: Zadkhosh SM, Zandi HG, Hemayattalab R. Neurofeedback versus mindfulness on young football players' anxiety and performance. Turk J Kinesiol, 2018; 4(4): 132-141.

$凶$ S. M. Zadkhosh, e-mail: mohamadzadkhosh@gmail.com DOI: 10.31459/turkjkin.467470 
According to Cashmore (2008), the term "anxiety" returns to unpleasant emotional that ambiguously characterized by fear and persistent panic. During the exercise performance, individual errors, unlucky, insult or exceptional performance of rivals are the most common sources of stress (Anshel \& Wells, 2000). Interpersonal issues often can effect on athletes' exercise performance, such as depression, substance use, gambling and eating disorder. Negative emotions resulting from interpersonal relationship crises such as divorce, loss of a dear and bad relations with parents also reduce performance. The athlete-coach relationship is a source of acute stress (Fletcher \& Hanton, 2003). Especially when this relationship turned out to be a conflict between the two sides. Some other issues related to the athletic environment such as role ambiguity on the team, leading to anxiety and stress in athletes (Beauchamp et al., 2003). Some issues could also lead to stress, such as numerous trips to competition (Waterhouse et al., 2004), team selection and financial support (Fletcher \& Hanton, 2003). Therefore, try to teach ways to reduce stress in sport is defined as the main tasks of sport psychologists.

Mindfulness and Neurofeedback interventions are included interventions that are used in the field of psychology, particularly sport psychology that includes two disparate aspects of psychological interventions i.e. technological and traditional. Neurofeedback with advanced and modern equipment emerged in performance promotion field and Mindfulness also derived from traditional rituals of Buddhism in Asia. Neurofeedback is a brain intervention that by using a computer allows occur self-assessment of brain waves. This is completed by stimulating visual and aural feedback through EEG activated rhythms of brain real time shows, subsequently used by individuals to selfassessment of EEG waves. As well as, according to the findings Ros et al. (2009) have reported that those who received neurofeedback training their appropriate ratio of brain waves were associated with effective function and reducing anxiety, on the other hand, eastern traditional Mindfulness meditation recently has emerged in psychology as an increasingly popular approach to improve wellbeing. Actually, many studies have been recorded benefits of Mindfulness meditation training to reduce depression, anxiety and stress both clinical (Hofmann et al., 2010) as well as non-clinical samples (Eberth \& Sedlmeier, 2012).
The use of neurofeedback history dates back to the 1970s. Neurofeedback is safe and non-invasive during which, sensors called electrodes are attached to the surface of the patient's head (Kaiser \& Othmer, 2000). These sensors record data's of level of brain activity and shows them in the form of brainwaves. This method uses self-assessment to set brain waves. In this method, an electrode located in a particular region of the head (with respect to corrective action that is being performed) two electrodes are attached to both ears. Electrode receives the desired wave and bring to a computer. At this time, the individual through the speaker or on the screen can received changes of brain waves into audio or video feedback. Actually stimulated brain wave activities. Often these simulations are like playing movies, music or video games. It should be noted that tools used during neurofeedback (electrodes and records the activities device), are used merely as a source record and provide information and did not invasive aspect. Neurofeedback likened to a mirror can be helpful in explaining the process of its effect. When wound occur on an individual face, the person with the scar in the mirror, understand the amount and depth of the wound and bandaging it. As well as, in neurofeedback person observes their abnormal brain waves and dispel them. Researches show that neurofeedback is an effective method to treat various disorders including anxiety, epilepsy and ADHD as well as increased performance (Arns et al., 2009). As Sterman (2000) has stated, neurofeedback applications were helpful particularly in the treatment of paroxysmal disorders in patient who do not respond to drug treatments. After extensive early studies, the next studies examine the effectiveness of neurofeedback was pulled in increasing the performance such exercise (Jones et al., 2007).

The use of neurofeedback in sports environments has many applications. Learning psychological state regulation before and during the competition can have a very positive effect on athletic performance (Hanin, 2003). Control and regulate arousal level for athletes can mean success or failure in an athletic event (Deeny et al., 2003). Set the range of optimal arousal is a difficult skill and usually it is shaped over time and with practice. However, even the most skilled athletes often are vulnerable and at risk when stress becomes too much (Hanin, 2003). In the meantime different methods of biofeedback has been able to help athletes in order to adjust the level of arousal, stress 
management and improve their performance. Most studies in sports psychology and neurofeedback are mostly associated with emotion regulation, excitement, concentration and their relationship to performance (Ruiz et al., 2017). Setting this arousal and concentration state has been recognized as a critical aspect of performing with excellence. In the past, studies have been conducted on elite athletes that have shown different patterns of EEG to amateur athletes (Crews \& Landers, 1993). Other studies of neurofeedback usage have similar findings in the field of sport (Crews \& Landers, 1993; Landers et al., 1991). Landers et al. (1991) in a study on the archers and shooters in moderate level found that neurofeedback training can be an effective tool in improving the positive performance of moderate athletes. Dupee (2008) shows that neurofeedback training can effect on cognitive skills of skiers such as attention and anxiety and athletes reported their mental states improve and increase in self-confidence, but were not observed changes in their athletic scores.

Mindfulness training developed over thousands of years and originated of Eastern philosophy (Kabat-Zinn, 2005). Interventions based on mindfulness are considered as a third-generation cognitive-behavioural therapies or third wave. Mindfulness is a form of meditation that rooted in the teachings of East religious and Buddha in particular (Öst, 2008). In Mindfulness meditation there is a form of relaxation with attention control so that the effect of each of the attention ranges, beliefs, expectations, mood, and emotion regulation affect sensory processes (Zeidan et al., 2012). In this intervention the person tries to focus his full attention to the present moment, so that with full acceptance and non-judgment. The researches at the same time have been considered so that in mindfulness studies and interventions has been used in a variety of normal and clinical populations. Over the past forty years, mindfulness developed in the field of applied clinical psychology, along with early intervention focused on traditional mindfulness meditation training. Mindfulness interventions significantly have been useful in populations with chronic pain and stress (KabatZinn et al.,), depression (Khoury et al., 2013) and anxiety (Roemer et al., 2008). These interventions have focused on the orientation of the attention of people to experience present and engage in nonjudgmental cognitive awareness of inner and outer and physical experiences in every moment (KabatZinn, 2005) to develop a new and neutral way to thoughts, feelings and emotions (Bernier et al., 2009).

Mindfulness has important concepts in sport psychology. This new way of study in applied psychology developed during the last ten years and recently have been focused on the center of mindfulness intervention and performance improvement of professional athletes. Now, theoretically interventions are in the intermediate step of sport psychology literature. Mindfulness in enhancing athletic performance has focused on mental skills training and optimal performance (Moore, 2009). A growing number of studies that have shown this intervention in sport will be considered later. With the development intervention as acceptance and commitment Mindfulness, Gardner \& Moore (2004) were the first to introduce Mindfulness research in sports psychology as a way to enhance athletic performance. Gardner \& Moore contrast to the traditional approach in sport psychology interventions by identifying the importance acceptance present experiences rather than targeting modify the content and were used on an acceptance Commitment Therapy model (Hayes \& Wilson, 2003) to develop their approach. They claimed that traditional approaches have targeted negative unwanted threads in consciousness. Only a few number of empirical studies have been done on the acceptance and commitment Mindfulness protocol, but the results suggested that this approach may effectively enhance performance (Schwanhausser, 2009). Other interventions of mindfulness have been developed in sport with some positive effects (Bernier et al., 2009).

Considering the many interventions in the field of sport psychology and the effect of anxiety on athletic performance, the present study was conducted to answer the question whether two active interventions of Mindfulness and neurofeedback $\mathrm{A} / \mathrm{T}$ protocol compared to a control group had an effect on the reduction of symptoms of anxiety and as a result, increase the performance of athletes? And if they have an impact, which one is most appropriate for the use of sports psychologists during intervention sessions?

\section{Methods}

\section{Participation}

The population of this research study included 45 football players aged between 17 and 20 years of 
Khorasan Razavi province that located in the north east of Iran in the national league was formed in 2016. Samples were enrolled based on paralleling and on the basis of pre-test score scale shooting performance in three groups of 15 subjects, experimental 1 (intervention based on alpha/theta neurofeedback training), experimental 2 (intervention based on mindfulness meditation) and control group. The first experimental group by neurofeedback alpha/theta and second experimental group were trained by the Mindfulness intervention for sports and the control group did not receive any intervention.

\section{Instrumentation}

\section{Sport Anxiety Scale}

Sports Anxiety Scale (Smith et al., 1990) is a multidimensional self-report test that is made to measure physical and cognitive anxiety. The test contains 21 statements of three subscales. This following measures include physical anxiety (9 items), worry ( 7 items) and decentralization (5 items). The participants will be asked to determine before the start of competition or during do it, to what extent, feelings and thoughts expressed in terms of test experience. The findings suggest that this test is reliable and valid. Smith et al. (1990) had been implemented retest reliability of the scale on which 77 football players (after 18 days), have reported 0.77 . In another study, also obtained higher scale of 0.85 retest reliability (after 7 days).

\section{Football Skill Shooting Test}

In order to evaluate athletic performance were used by Christine Moore shooting skills test to detect the condition to determine the state in the pre-test and amount of progress in post-test. Subjects in the starting line (16-meter) placed and shoot the ball towards the target fixed. Subjects use their superior foot. The ball can be arbitrary at any point of the line's shoot. Subjects first will do four shooting to prepare. Subjects do test in four phases and each phase four shoots (A total of 16 Shoot knocks). The way that scores to players is based on their shoot, if a player wants shoot into the top right side and hit to the spot, he scores 10 points and if shoot to the bottom right he scores 4 points. The shoots were not scored if to be rolling on the floor. The final score achieved based on 16 times shooting (Mor \& Christian, 1979).

\section{Neurofeedback}

Neurofeedback is a comprehensive treatment system that works directly with the brain. Neurofeedback device, is a tool that analyses raw brain waves received through electrodes placed on the head to the frequency of the delta, theta, alpha and beta. Neurofeedback training methods using devices equipped with Procomp computer system and conducted by researchers monitoring. These tools include BioGraph hardware and Thought Technology Ltd company software. This device was composed of a pair of electrodes that were placed on the head, according to the international 10-20 system.

\section{Procedure}

Samples based on matching and on the basis of pretest shooting performance scores divided into two groups, groups 1 (alpha / theta neurofeedback intervention protocols) and group 2 (mindfulnessbased intervention). In both groups before starting the project were investigated by Sport Anxiety Scale questionnaire to assess the level of sport anxiety and Moore - Christian shooting performance test (Fig. 1) to assess the level of the athlete's performance. Then the interventions were performed in experimental groups as follows.

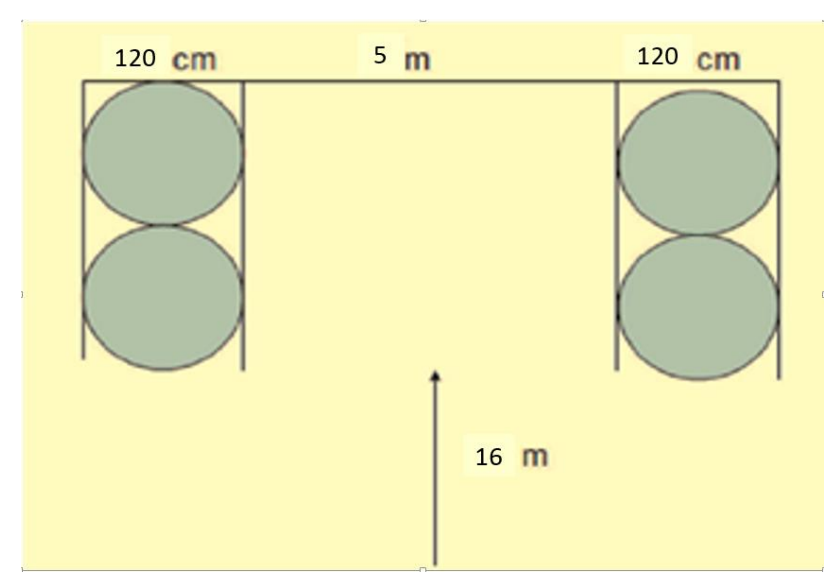

Figure 1. Moore - Christian shooting performance test.

\section{Neurofeedback Group}

In this group athletes attended over a period of neurofeedback training in the form of theta increase/ alpha reduction in the area of $\mathrm{Pz}$ (located in the parietal cortex area) during 6 weeks for two 30-minute sessions per week a total of twelve sessions. In each neurofeedback session every person focused on the reduction of alpha wave and 
increase theta waves. Alpha frequency in the range of 8 to $11 \mathrm{~Hz}$ and theta frequency in the range of 5 to $8 \mathrm{~Hz}$ were determined. In the first session was described to participants to explain the connection between neurofeedback devices, athlete's body, computer and voice feedback. It was explained to participants that have enough sleep the night before neurofeedback training in during the session do not sleep due to fatigue and sleepiness during the meeting. As well as a description for teaching mental imagery that was used during the intervention sessions were presented to the participants. Participants were guided to sit on the armchair with his eyes closed and during the session pay mental imagery. Active electrode has been prepared by using Nuprep gel, ear cleaner and hygienically clean cotton in the Pz region and by ten 20 adhesive was connected at the desired location with reference electrode in the left ear and right ear electrode Grande. This protocol was used in acoustic feedback that the subjects by closing their eyes responded to the sound waves that were spread to him, these sounds include the sound of ocean waves and Roaring River. At the beginning of each session, participants were guided to sit comfort and quiet on a chair and by examiners command closes his eyes and the session was started. While that should have been prevented from sleeping subject. When the person went to sleep mode by increasing his delta wave the device with the sound of crickets that was set to inform the examiner were appraised and he was prevented from sleeping. Participants At the end of the session reviewed the session with the examiner (Gruzelier, 2014).

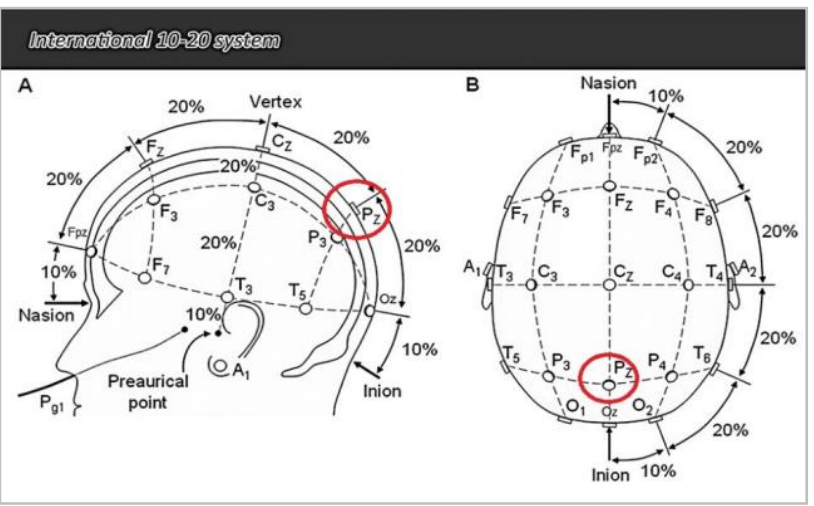

Figure 2. PZ area in the international system 10-20.

\section{Neurofeedback Group}

Athletes in this group received twelve sessions of mindfulness meditation training for sport, over six weeks for two 30-minute sessions per week (Baltzell \& Akhtar, 2014). A program entitled "mental training through meditation" was introduced to athletes and coaches because may they don't know about meditation and our purpose was to be created common mental structures. In general, the primary purpose of the sessions was to increase mindfulness and then to train a mindfulness skill during practice and competition. The training was similar to Jon Kabat Zin's trainings, in that training the focus of meditation leader was on mindfulness meditation practice and participants were encouraged to practice daily. In addition, athletes while practicing accepting and non-judgmental thoughts and feelings. 20 minutes of each session were assigned to educate participants on different aspects of mindfulness, the training mindfulness meditation and further questions by educator after meditation practice. The educator was assigned a time per session for participants (athletes and coaches) to ask questions. 10 minutes of each session were devoted to meditation practice. There were four main areas to practice: Open awareness, capacity, Caring thoughts for self and teammates, Concentration exercises and practicing acceptance of negative mind-states. After each session with a discussion of how the skills learned at the meditation training could transfer directly to the training field or competition were concluded.

\section{Results}

During the study period, the three subjects of the neurofeedback group, three of the mindfulness group and three of the control group were excluded of study for reasons interrupted cooperation and not complete interventions and training courses. As shown in Table 1, the mean values of the twophases, pre-test and post-test were different, so that the mean performance in groups rising and decreasing levels of anxiety scores in groups. Then, to evaluate the significance of these changes in different groups, Mixed ANOVA and the results are reported in Table 2.

According to Table 2, it can be argued that the main effects of the interventions were cause of significant changes in the levels of performance and sport anxiety. As shown in Table 2, the main effects of the interventions were significant on anxiety and performance levels of athletes and interactive intervention effect in both intervention groups was significant for the athlete's performance and anxiety levels. Then, in Table 4, which represents the 
difference means mindfulness and neurofeedback groups in the pre-test and post-test, it was found that with the passage of time and intervention in the sport anxiety and performance levels in both experimental groups was made more significant difference than the control group. On the other hand, in mindfulness group the level of sport anxiety decreased more than neurofeedback group.

Table 1

Descriptive statistics (Mean \pm SD).

\begin{tabular}{llccc}
\hline Variable & Group & Pre-test & Post-test & Difference \\
\hline Performance & Neurofeedback & $58.66 \pm 37.41$ & $76.66 \pm 40.50$ & 18.00 \\
& Mindfulness & $70.33 \pm 30.82$ & $60.16 \pm 35.55$ & 19.833 \\
& Control & $58.33 \pm 31.27$ & $69.16 \pm 26.86$ & 10.83 \\
Sport Anxiety & Neurofeedback & $51.00 \pm 14.10$ & $47.66 \pm 14.20$ & -3.334 \\
& Mindfulness & $53.33 \pm 12.04$ & $46.00 \pm 10.12$ & -7.333 \\
& Control & $47.00 \pm 16.07$ & $44.83 \pm 14.04$ & -2.17 \\
\hline
\end{tabular}

Table 2

Tests of within-subjects contrasts.

\begin{tabular}{|c|c|c|c|c|c|c|c|}
\hline & Source & Type III Sum of Squares & $\mathrm{df}$ & Mean Square & $\mathrm{F}$ & $\mathrm{p}$ & Partial $\eta^{2}$ \\
\hline \multirow[t]{3}{*}{ Performance } & Factor1 & 9473.778 & 1 & 9473.778 & 63.449 & .000 & .658 \\
\hline & Factor $1 *$ Group & 542.889 & 2 & 271.444 & 1.818 & .178 & .099 \\
\hline & Error(factor1) & 4927.333 & 33 & 149.313 & & & \\
\hline \multirow[t]{3}{*}{ Sport Anxiety } & Factor1 & 658.778 & 1 & 658.778 & 37.035 & .000 & .529 \\
\hline & Factor $1 *$ Group & 176.222 & 2 & 88.111 & 4.953 & .013 & .231 \\
\hline & Error(factor1) & 587.000 & 33 & 17.788 & & & \\
\hline
\end{tabular}

Table 3

Tests of between-subjects effects.

\begin{tabular}{llcccccc}
\hline & Source & Type III Sum of Squares & df & Mean Square & F & p & Partial $\eta^{2}$ \\
\hline Performance & Intercept & 179211.111 & 1 & 179211.111 & 159.746 & .000 & .829 \\
& Group & 1783.722 & 2 & 891.861 & .795 & .460 & .046 \\
& Error & 37021.167 & 33 & 1121.854 & & & \\
Sport Anxiety & Intercept & 84003.361 & 1 & 84003.361 & 467.887 & .001 & .944 \\
& Group & 103.389 & 2 & 51.694 & .288 & .752 & .017 \\
& Error & 5924.750 & 33 & 179.538 & & & \\
\hline
\end{tabular}

Table 4

Results of ANOVA with repeated measures.

\begin{tabular}{lcccccc}
\hline Source & Sum of Squares & df & Mean Square & F & p & Partial $\eta^{2}$ \\
\hline Performance & 1783.722 & 2 & 891.861 & .795 & .460 & .046 \\
Error & 37021.167 & 33 & 1121.854 & & & \\
Sport Anxiety & 103.389 & 2 & 51.694 & .288 & .752 & .017 \\
Error & 5924.750 & 33 & 179.538 & & & \\
\hline
\end{tabular}




\section{Discussion}

The aim of this study was to investigate the effects of neurofeedback and mindfulness training on performance and anxiety of young football players of Khorasan Razavi province that presented in 2016 Iranian national league. For this purpose, 45 football players who were attended in national football league of Iran were assigned to three experimental groups. Athletes in experimental group 1 received twelve-session, 30-minute alpha/theta neurofeedback training, in experimental group 2 received twelve-session, and 30-minute mindfulness meditation training and control group did not receive any intervention. Before and after interventions sport anxiety scale questionnaire and shoot performance test were used to collect data. The results of MANOVA showed that the improvement of athletic performance scores and reduction of anxiety scale were significant in the neurofeedback group rather than the control group. In addition, the results of the mindfulness group showed that the improvement of athletic performance scores and reduction of anxiety scale scores were more significant rather than control group. And there were significant different between mindfulness group compared to the neurofeedback group with sport anxiety scale, but there were not significant differences between the neurofeedback group compared to the mindfulness group in athletic performance.

During the competitive level athletes are always trying to have a high level of performance. Injury, not select in a team and low progress of technical skills may hinder their better performance; in addition, psychological issues often affect athletic performance. Advances in sports psychology researches have revealed psychological skills as a key factor in athletic performance. Most professional athletes have begun exercise at a very young age as a special exercise has developed their skills over time, so they do psychological skills. Now sport organizations focused on the importance of the development of psychological skills in young elite athletes. Learning the basic skills may help further their athletic performance at a high level to overcome the emotional needs through adaptive ways to prepare. One of the most important factors that strongly effects on the performance of athletes, especially in certain critical situations is anxiety (Jamshidi et al., 2009). Since there are many ways to reduce anxiety and increase athletic performance, with the presence of technologies in the world in the field of sports psychology alongside traditional methods of problem arises as to which method is more effective for anxiety and athletic performance.

Review the data of the study showed that mindfulness training have positive effects on athletic performance and anxiety and increase performance and reduce anxiety of football players. In parallel to these results has been done researches that can point out to Kee et al. (2012) that investigated the effects of 9 weeks mindfulness training on 32 men with an average age of $22 / 8 \pm$ $1 / 94$ and the results indicated a positive influence of mindfulness induction on motor control and balance the performance of the experimental group compared to the control group. In another study, Thompson et al. (2011) in their study on the performance of 25 athletes examined the athlete's performance after attending four weeks and follow up them mindfulness training. The results indicated performance improvements of athletes from pre-test to follow up. Kerr et al. (2011) also in their study investigated the effect of eight weeks of mindfulness training on 16 men and women with an average age of $31 / 6$ that results show significant improvements of participants' spatial attention. Other studies have been conducted by mindfulness trainings to improve athletic performance that can point out the researches of (Bernier et al., 2009; Kabat-Zinn et al. (1985) in one of the first studies in the field of sport investigated the effect of mindfulness meditation training on collegiate and Olympic rowers that results showed improvement of athletic performance.

But in contrast with obtaining results have been conducted some researches. For example, can pointed out to Call et al. (2014) that examined the effects of mindfulness training on 91 undergraduate women to reduce stress and anxiety symptoms within three weeks of 45-minute that the results showed symptoms of stress and anxiety. As well as Roemer et al. (2008) have shown the effects of mindfulness training on stress reduction. In the field of sport also Scott-Hamilton, Schutte, and Brown (2016) examined the effects of eight weeks of mindfulness training on sport anxiety, pessimism and flow on competitive cyclists. In this study that was conducted on 47 cyclist and the results showed an anxiety reduction and facilitate the psychological experiences of athletes. 
As well as study reviews in the field of neurofeedback interventions showed that neurofeedback training have a positive effect on the performance and enhances athletic performance. Other studies have shown that neurofeedback training in other fields also increased performance. In five weeks of alpha / theta neurofeedback training by Raymond et al. (2005) on 24 female and male ballerinas on university level, the results showed an increase in performance of the experimental group compared to the control group. In another study by Leach et al. (2008) examined the ability of musicians, from beginner level by neurofeedback interventions that participants were randomly assigned to three groups: alpha / theta, SMR and control. The results indicate an increase in the alpha / theta and SMR groups compared with the control. On the other hand, Ros et al. (2009) examined the surgeon's performance by alpha / theta neurofeedback; that the results were inconsistent with other studies and show that alpha / theta training was accompanied by an increase surgeons' performance. The review of Studies conducted in the field of sports shows that one of the first neurofeedback interventions to enhance athletic performance was Landers et al. (1991) study that examined archery performance. The results of this study show that correct feedback by neurofeedback lead to improve archers performance while incorrect feedback lead to reducing their performance. In another case study by Graczyk et al. (2014) on the Olympic athletes after injury results indicate increase athletic performance after neurofeedback training. Also, Shaw et al., (2012) showed increased balance and performance of gymnasts in the competition after neurofeedback training. In parallel with the results obtained in this study, other studies in the field of sport show that neurofeedback can facilitate athletic performance (Landers et al., 1991; Rostami, Sadeghi, Karami, Abadi, \& Salamati, 2012). Thus, it seems that the use of alpha / theta neurofeedback interventions directly result to football player performance enhancement.

On the other hand, analysis of the results indicated that alpha / theta neurofeedback training cause to sport anxiety reduction. In this regard, studies in the fields of sports and non-sports demonstrate similar results, which reflects the reduction in anxiety as a result of alpha / theta neurofeedback training. In the study by Schwartz \& Andrasik (2017) neurofeedback training showed a positive effect on the treatment of anxiety disorders. In another study conducted on medical students with high social anxiety, 12 medical students randomly assigned to alpha / theta training groups, sham and control groups. Neurofeedback training was 10 sessions, twice a week and the results indicated an increase in pluripotency, confidence, composure and temper in the alpha / theta neurofeedback group (Raymond et al., 2005), but on the other hand, some studies showed no effect of neurofeedback on some features. For example, Raymond et al. (2005) in their study of 12 participants during the ninth session of alpha / theta neurofeedback protocol training showed that the trainings are not enough to change the character, but to promote temper demonstrated positive results.

The overall results show that mindfulness training and $\mathrm{A} / \mathrm{T}$ neurofeedback are effective trainings to do as sport psychology interventions and compare two interventions shows that neurofeedback and mindfulness are effective to increase performance and reduce anxiety of football players. The results also indicate that the effect of mindfulness on anxiety reduction of football players is more in comparison with neurofeedback. Therefore sport psychologists can use this technique in conjunction with other interventions to improve the performance and reduce the anxiety of their athletes.

\section{References}

Anshel MH, Wells B. Sources of acute stress and coping styles in competitive sport. Anxiety, stress and coping, 2000; 13(1): 1-26.

Anshel MH, Williams L, Williams SM. Coping style following acute stress in competitive sport. The Journal of Social Psychology, 2000; 140(6): 751-773.

Arns M, de Ridder S, Strehl U, Breteler M, Coenen A. Efficacy of neurofeedback treatment in ADHD: the effects on inattention, impulsivity and hyperactivity: a meta-analysis. Clinical EEG and Neuroscience, 2009; 40(3): 180-189.

Baltzell A, Akhtar VL. Mindfulness meditation training for sport (MMTS) intervention: Impact of MMTS with division I female athletes. Journal of Happiness and Well-being, 2014; 2(2): 160-173.

Beauchamp MR, Bray SR, Eys MA, Carron AV. The effect of role ambiguity on competitive state anxiety. Journal of Sport and Exercise Psychology, 2003; 25(1): 77-92.

Bernier M, Thienot E, Codron R, Fournier JF. Mindfulness and acceptance approaches in sport performance. 
Journal of Clinical Sport Psychology, 2009; 3(4): 320-333.

Call D, Miron L, Orcutt H. Effectiveness of brief mindfulness techniques in reducing symptoms of anxiety and stress. Mindfulness, 2014; 5(6): 658668.

Cashmore E. Sport and exercise psychology: the key concepts: Routledge, 2008.

Crews DJ, Landers DM. Electroencephalographic measures of attentional patterns prior to the golf putt. Medicine \& Science in Sports \& Exercise, 1993.

Deeny SP, Hillman CH, Janelle CM, Hatfield BD. Corticocortical communication and superior performance in skilled marksmen: An EEG coherence analysis. Journal of Sport and Exercise Psychology, 2003; 25(2): 188-204.

Dupee M. Exploring a bioneurofeedback training intervention to enhance psychological skills \& performance in sport. University of Ottawa (Canada), 2008.

Eberth J, Sedlmeier P. The effects of mindfulness meditation: A meta-analysis. Mindfulness, 2012; 3(3): 174-189.

Fletcher D, Hanton S. Sources of organizational stress in elite sports performers. The Sport Psychologist, 2003; 17(2): 175-195.

Gardner FL, Moore ZE. A mindfulness-acceptancecommitment-based approach to athletic performance enhancement: Theoretical considerations. Behavior Therapy, 2004; 35(4): 707723.

Gould D, Dieffenbach K, Moffett A. Psychological characteristics and their development in Olympic champions. Journal of Applied Sport Psychology, 2002; 14(3): 172-204.

Graczyk M, Pachalska M, Ziolkowski A, Manko G, Lukaszewska B, Kochanowicz K, Mirski A, Kropotow I. Neurofeedback training for peak performance. Annals of Agricultural and Environmental Medicine, 2014; 21(4): 871-875.

Gruzelier JH. EEG-neurofeedback for optimising performance. II: creativity, the performing arts and ecological validity. Neuroscience \& Biobehavioral Reviews, 2014; 44: 142-158.

Hanin YL. Performance related emotional states in sport: a qualitative analysis. Paper presented at the Forum Qualitative Sozialforschung/Forum: Qualitative Social Research, 2003.

Hayes SC, Wilson KG. Mindfulness: Method and process. Clinical Psychology: Science and Practice, 2003; 10(2): 161-165.
Hofmann SG, Sawyer AT, Witt AA, Oh D. The effect of mindfulness-based therapy on anxiety and depression: A meta-analytic review. Journal of Consulting and Clinical Psychology, 2010; 78(2): 169.

Jamshidi A, Bagherzadeh F, Arab Ameri E, Rastegar A. Compare sports orientation athletes participating in the eighth Olympiad student sports in Iran. Journal of Harekat, 2009; 38: 39-52.

Jones G, Hanton S, Connaughton D. A framework of mental toughness in the world's best performers. The Sport Psychologist, 2007; 21(2): 243-264.

Kabat-Zinn J. Coming to our senses: Healing ourselves and the world through mindfulness: Hachette UK, 2005.

Kabat-Zinn J, Lipworth L, Burney R. The clinical use of mindfulness meditation for the self-regulation of chronic pain. Journal of Behavioral Medicine, 1985; 8(2): 163-190.

Kaiser DA, Othmer S. Effect of neurofeedback on variables of attention in a large multi-center trial. Journal of Neurotherapy, 2000; 4(1): 5-15.

Kee YH, Chatzisarantis NN, Kong PW, Chow JY, Chen LH. Mindfulness, movement control, and attentional focus strategies: effects of mindfulness on a postural balance task. Journal of Sport and Exercise Psychology, 2012; 34(5): 561-579.

Kerr CE, Jones SR, Wan Q, Pritchett DL, Wasserman RH, Wexler A, Villanueva JJ, Shaw JR, Lazar SW, Kaptchuk TJ, Littenberg R, Hämäläinen MS, Moore CI. Effects of mindfulness meditation training on anticipatory alpha modulation in primary somatosensory cortex. Brain Research Bulletin, 2011; 85(3): 96-103.

Khoury B, Lecomte T, Fortin G, Masse M, Therien P, Bouchard V, Chapleau MA, Paquin K, Hofmann SG. Mindfulness-based therapy: a comprehensive meta-analysis. Clinical Psychology Review, 2013; 33(6): 763-771.

Krane V, Williams J. Psychological characteristics of peak performance. In: Applied sport psychology: Personal growth to peak performance (Editor: J. M. Williams, 5. Edition, New York: McGraw-Hill, 2006: 207-227.

Landers DM, Petruzzello SJ, Salazar W, Crews DJ, Kubitz KA, Gannon TL, Han M. The influence of electrocortical biofeedback on performance in preelite archers. Medicine \& Science in Sports \& Exercise, 1991; 23(1):123-129.

Leach J, Holmes P, Hirst L, Gruzelier J. Alpha theta versus SMR training for novice singers/advanced instrumentalists. Revista Espanola Psichologica, 2008; 10: 62. 
Martin GL, Vause T, Schwartzman L. Experimental studies of psychological interventions with athletes in competitions: Why so few? Behavior Modification, 2005; 29(4): 616-641.

Moore ZE. Theoretical and empirical developments of the Mindfulness-Acceptance-Commitment (MAC) approach to performance enhancement. Journal of Clinical Sport Psychology, 2009; 3(4): 291-302.

Mor D, Christian V. The development of a skill test battery to measure general soccer ability. North Carolina Journal of Health and Physical Education, 1979; 15(1): 30.

Orlick T, Partington J. Mental links to excellence. The Sport Psychologist, 1988; 2(2): 105-130.

Öst LG. Efficacy of the third wave of behavioral therapies: A systematic review and meta-analysis. Behaviour Research and Therapy, 2008; 46(3): 296-321.

Raymond J, Varney C, Parkinson LA, Gruzelier JH. The effects of alpha/theta neurofeedback on personality and mood. Cognitive brain research, 2005; 23(2): 287-292.

Roemer L, Orsillo SM, Salters-Pedneault K. Efficacy of an acceptance-based behavior therapy for generalized anxiety disorder: evaluation in a randomized controlled trial. Journal of Consulting and Clinical Psychology, 2008; 76(6): 1083.

Ros T, Moseley MJ, Bloom PA, Benjamin L, Parkinson LA, Gruzelier JH. Optimizing microsurgical skills with EEG neurofeedback. BMC neuroscience, 2009; 10(1): 87 .

Rostami R, Sadeghi H, Karami KA, Abadi MN, Salamati $P$. The effects of neurofeedback on the improvement of rifle shooters' performance. Journal of Neurotherapy, 2012; 16(4): 264-269.

Ruiz MC, Raglin JS, Hanin YL. The individual zones of optimal functioning (IZOF) model (1978-2014): historical overview of its development and use.
International Journal of Sport and Exercise Psychology, 2017; 15(1): 41-63.

Schwanhausser L. Application of the mindfulnessacceptance-commitment (MAC) protocol with an adolescent springboard diver. Journal of Clinical Sport Psychology, 2009; 3(4), 377-395.

Schwartz MS, Andrasik F. Biofeedback: A practitioner's guide: Guilford Publications, 2016.

Scott-Hamilton J, Schutte NS, Brown RF. Effects of a mindfulness intervention on sports-anxiety, pessimism, and flow in competitive cyclists. Applied Psychology: Health and Well-Being, 2016; 8(1): 85-103.

Shaw L, Zaichkowsky L, Wilson V. Setting the balance: Using biofeedback and neurofeedback with gymnasts. Journal of Clinical Sport Psychology, 2012; 6(1): 47-66.

Smith RE, Smoll FL, Schutz RW. Measurement and correlates of sport-specific cognitive and somatic trait anxiety: The Sport Anxiety Scale. Anxiety Research, 1990; 2(4): 263-280.

Sterman MB. Basic concepts and clinical findings in the treatment of seizure disorders with EEG operant conditioning. Clinical Electroencephalography, 2000; 31(1): 45-55.

Thompson RW, Kaufman KA, De Petrillo LA, Glass CR, Arnkoff DB. One year follow-up of mindful sport performance enhancement (MSPE) with archers, golfers, and runners. Journal of Clinical Sport Psychology, 2011; 5(2): 99-116.

Waterhouse J, Reilly T, Edwards B. The stress of travel. Journal of Sports Sciences, 2004; 22(10): 946-966.

Zeidan F, Grant J, Brown C, McHaffie J, Coghill R. Mindfulness meditation-related pain relief: evidence for unique brain mechanisms in the regulation of pain. Neuroscience Letters, 2012; 520(2): 165-173. 